\title{
Low-temperature acoustic characteristics of the amorphous alloy $\mathrm{Zr}_{41.2} \mathrm{Ti}_{13.8} \mathrm{Cu}_{12.5} \mathrm{Ni}_{10} \mathrm{Be}_{22.5}$
}

\author{
A. L. Gaiduk, E. V. Bezuglyi, and V. D. Fil \\ B. Verkin Institute for Low Temperature Physics and Engineering, National Academy of Sciences \\ of the Ukraine, 310164 Kharkov, Ukraine*
}

W. L. Johnson

California Institute of Technology, Pasadena, CA 91125, USA

(Submitted July 22, 1997)

Fiz. Nizk. Temp. 23, 1139-1143 (October 1997)

The temperature dependences of the sound velocity $v$ and attenuation $\alpha$ of high-frequency $(50-160 \mathrm{MHz})$ sound in the bulk amorphous alloy $\mathrm{Zr}_{41.2} \mathrm{Ti}_{13.8} \mathrm{Cu}_{12.5} \mathrm{Ni}_{10} \mathrm{Be}_{22.5}$ are studied at helium temperatures in the normal and superconducting states. The alloy is characterized by a relatively small constant $C$ determining the intensity of interaction between an elastic wave and two-level systems. The density of states of the latter systems is estimated. The peculiarities in the variation of $v$ during the superconducting transition point to the possibility of a gapless superconductivity in a narrow temperature interval near $T_{c}$. () 1997 American Institute of Physics. [S1063-777X(97)01810-0]

Low-temperature kinetics of amorphous materials is mainly determined by specific low-energy quasilocal excitations, viz., two-level systems (TLS). The model concept of TLS developed in Refs. 1 and 2 proved to be very fruitful for explaining the thermal, elastic, and electromagnetic properties of insulating glasses and metglasses. ${ }^{3}$ A simplest model of a TLS is a heavy tunneling entity in an asymmetric double-well potential. Both the initial model of TLS and its subsequent versions ${ }^{4}$ are universal and are not connected with specific features associated with the microscopic origin of double-well potentials.

The properties of a large number of various glasses have been studied extensively. One of the most remarkable results in this field is the conclusion concerning virtual independence of the TLS density of states of the composition and the method of obtaining of glass. As regards metglasses, it should be noted, however, that all the compositions that were investigated before had been obtained at very high cooling rates $\left(\geqslant 10^{3} \mathrm{~K} / \mathrm{s}\right)$, which probably determines the abovementioned universality.

New classes of glass-forming systems obtained recently are stable over a wide temperature region of supercooled liquid state. Their high resistance to crystallization makes it possible to obtain bulk amorphous samples at very low quenching rates $(\leqslant 10 \mathrm{~K} / \mathrm{s})$. It would be interesting to estimate the density of states of TLS for a typical representative of this family of metglasses. In this connection, we studied the acoustic properties of the amorphous alloy $\mathrm{Zr}_{41.2} \mathrm{Ti}_{13.8} \mathrm{Cu}_{12.5} \mathrm{Ni}_{10} \mathrm{Be}_{22.5}{ }^{5}$ at temperatures near $1 \mathrm{~K}$.

Acoustic measurements are known to be one of the most informative methods of studying TLS. ${ }^{3}$ The interaction of TLS with sound mainly follows the two principal mechanisms: resonance and relaxation. The former mechanism corresponds to the excitation of direct transitions between tun- nel levels with the energy splitting $E=\sqrt{\Delta^{2}+\Delta_{0}^{2}}=\omega$ by sound of frequency $\omega$ ( $\Delta$ is the asymmetry of a double-well potential and $\Delta_{0}$ the tunnel splitting of energy levels for $\Delta=0)$. In the available frequency range $(\omega \ll T)$, the resonant contribution to variations of the sound velocity is determined (to within a constant term) by the formula

$$
\left(\frac{\delta v_{i}}{v_{i}}\right)_{\text {res }}=\frac{\bar{P} \gamma_{i}^{2}}{\rho v_{i}^{2}} \ln T=C_{i} \ln T,
$$

where $\bar{P}$ is the TLS density of states, $\gamma$ the deformation potential, $\rho$ the mass density, and $i$ denotes the longitudinal $(l)$ or transverse $(t)$ polarization.

The resonant contribution to the absorption coefficient $\alpha / q$ ( $q$ is the wave number) is small in the parameter $\omega / T$ on the $C_{i}$ scale.

Elastic deformation of the wave also shifts the spectrum of tunnel levels. If there exist the relaxation mechanism which makes it possible for TLS to tune the occupancy of the levels to a new set of $E$ rapidly (on the $\omega^{-1}$ scale), the attenuation and the velocity of sound acquire a relaxation component:

$$
\left(\begin{array}{c}
\frac{\alpha_{i}}{q_{i}} \\
\frac{\delta v_{i}}{v_{i}}
\end{array}\right)=\frac{C_{i}}{2}\left(\begin{array}{c}
\pi \\
\ln \omega \tau_{\min }
\end{array}\right),
$$

where $\tau_{\min }$ is the minimum relaxation time for $\Delta=0$ and $E \sim T$. Expressions (2) are valid for $\omega \tau_{\min } \ll 1$; otherwise, the relaxation interaction can be neglected. In the general case, the TLS relaxation in metglasses is due to interaction with 


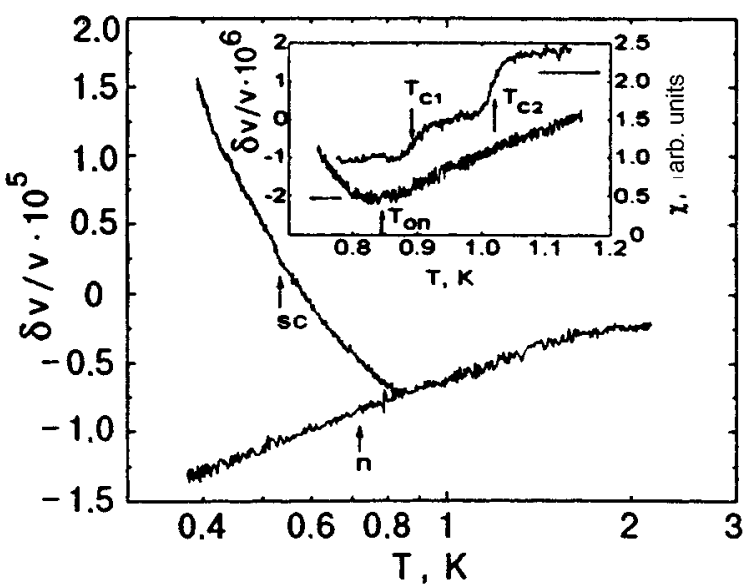

FIG. 1. emperature dependences of relative changes in the velocity of transverse sound in the amorphous alloy $\mathrm{Zr}_{41.2} \mathrm{Ti}_{13.8} \mathrm{Cu}_{12.5} \mathrm{Ni}_{10} \mathrm{Be}_{22.5}$ at a frequency of $54.3 \mathrm{MHz}$. The inset shows a fragment of $\delta v / v(T)$ dependence and the diamagnetic response $\chi$ in the superconducting transition region.

electrons as well as with phonons, but the contribution of the latter can be neglected at $T<2-3 \mathrm{~K}$, and the relaxation frequency can be written in the form ${ }^{3}$

$$
\left(\tau_{\min }^{-1}\right)_{e}=\frac{\pi}{2} \eta^{2} T,
$$

where $\eta$ is the dimensionless parameter of coupling between electrons and the TLS.

The sample with an acoustic path length of $0.74 \mathrm{~cm}$ was cut from an oval ingot. ${ }^{5}$ The values of $v$ and $\alpha$ were measured in the pulsed mode by the phase-sensitive bridge method at frequencies close to 54.3 and $162.9 \mathrm{MHz}$. The diamagnetic response $\chi$ was measured at a frequency of 22.5 $\mathrm{Hz}$ with the concentric arrangement of the sample, the receiving and exciting coils. Note the high homogeneity of the alloy: 8-10 well-defined reflexes were observed at an acoustic wavelength $\sim 15 \mu \mathrm{m}$. In order to avoid possible errors of interference origin, main measurements were made by comparing phases and amplitudes of first and second or third pulses. At $77 \mathrm{~K}$, the velocities were $v_{l}=(5.17 \pm 0.03)$ $\times 10^{5}, v_{t}=(2.49 \pm 0.01) \times 10^{5} \mathrm{~cm} / \mathrm{s}$.

Figure 1 shows the relative change in the velocity of transverse sound in the alloy under investigation in the normal $(n)$ and superconducting ( $s c)$ states. In the former case, the field $H=1.5 \mathrm{~T}(\mathbf{H} \| \mathbf{q})$ was applied. It should be noted that above $T_{c}$, such fields did not affect the behavior of $v$ and $\alpha$. In the normal phase, the temperature variation of the velocity is correctly approximated by a logarithmic dependence with the coefficient $C_{i} / 2$ appearing when expressions (1) and (2) are added. To within the error of measurements, the values of the constants $C_{i} \quad\left(C_{l}=(0.58 \pm 0.02) \times 10^{-5}, \quad C_{t}=(1.42\right.$ $\pm 0.03) \times 10^{-5}$ ) were independent of frequency.

In the superconducting phase, freezing out of normal excitations leads to an increase in $\tau_{\min }{ }^{6}$ and to a sharp decrease in the relaxation contributions to the velocity and absorption of sound, which is reflected in a sharp kink on the $v(T)$ curve in Fig. 1 at the temperature $T_{\text {on }}=0.85 \mathrm{~K}$. The inset to Fig. 1 shows the changes in the velocity near $T_{\text {on }}$ measured simultaneously $(H=0)$ with the diamagnetic re- sponse $\chi$ of the sample recorded for the ac field amplitude $\widetilde{H} \simeq 4$ mOe. The sample obviously contains two phases with the phase-transition temperatures $T_{c 1} \simeq 0.9 \mathrm{~K}$ and $T_{c 2} \simeq 1.02 \mathrm{~K}$. The value of $T_{c 2}$ probably corresponds to a surface phase since this transition does not affect the sound velocity in any way. Besides, an increase in $\widetilde{H}$ to $40 \mathrm{mOe}$ leads to virtually complete suppression of the jump in $\chi$ at $T_{c 2}$, while the peculiarity at $T_{c 1}$ is preserved up to $\widetilde{H}=1 \mathrm{Oe}$. The non-coincidence between the resistive and magnetic superconducting transitions in a glass of the same composition observed by Gerber et al. ${ }^{7}$ is apparently also connected with the existence of two phases.

It can be seen from Fig. 1 that the temperatures $T_{\text {on }}$ and $T_{c 1}$ do not coincide. Naturally, we can assume that the sample is not homogeneous as regards $T_{c 1}$, but the small width of the jump in $\chi$ at $T_{c 1}$ renders this assumption hardly plausible. Probably, the regime of so-called gapless superconductivity is realized in the temperature interval $T_{c 1}-T_{\text {on }} \cdot{ }^{8}$

An analytic expression for $\tau_{\min }$ in a superconductor can be derived only for $E \ll \Delta_{s c}$ ( $\Delta_{s c}$ is the superconducting gap). ${ }^{6}$ Since the main contribution to the measured parameters comes from TLS with $E \leqslant T$, the above limitation boils down to $T \ll \Delta_{s c}$. In this case, we can write

$$
\left(\tau_{\min }^{-1}\right)_{e s c}=\frac{\pi}{2} \eta^{2} T \mathrm{e}^{-\Delta_{s c} / T} .
$$

Since the velocity in the superconducting phase continues to grow up to $T \sim 0.4 \mathrm{~K}$, the condition $\omega \tau_{\min }<1$ obviously remains valid, and expression (2) for the relaxation contribution to $\delta v / v$ still holds. Assuming that the resonant contribution in the normal and superconducting phases is the same, we have the following expression for the difference in the velocities of sound in the $s c$ - and $n$-phases from (1-4):

$$
\frac{\Delta v}{v}=\left(\frac{\delta v}{v}\right)_{s c}-\left(\frac{\delta v}{v}\right)_{n} \sim-\frac{\Delta_{s c}}{T} .
$$

In actual practice, the strong inequality between $T$ and $\Delta_{s c}$ is not satisfied in our experiments. Nevertheless, we can expect that the experimental dependences $\Delta v / v\left(T^{-1}\right)$ become linear in a certain temperature interval $T<T_{c}$, where $\Delta_{s c}(T) \approx \Delta_{s c}(0)$.

A dependence of this type is shown in Fig. 2a. For comparison with theoretical dependences, the temperature scale in Fig. 2 is given in reduced form, and the superconducting transition temperature is assumed to be equal to $T_{\text {on }}$. The velocity scale is also normalized to $C_{t} / 2$. It was found that the dependence $\Delta v / v\left(T^{-1}\right)$ is close to linear in the entire temperature range below $T_{\text {on }}$, but its slope is twice the BCS value of $\Delta_{s c}(0)$. In all probability, such a behavior of $\Delta v / v\left(T^{-1}\right)$ is the result of joint operation of several temperature-dependent mechanisms. For comparison, Fig. 2a presents the results of complete calculation of $\Delta v / v$ for the BCS model ( $\eta=0.85$, see below) on the basis of equations from Ref. 6. The agreement between the theoretical and experimental dependences is obviously unsatisfactory over virtually the entire temperature range, although the scale of variation is reproduced correctly on the whole. The agreement can probably be improved in the vicinity of $T_{c}$ by 


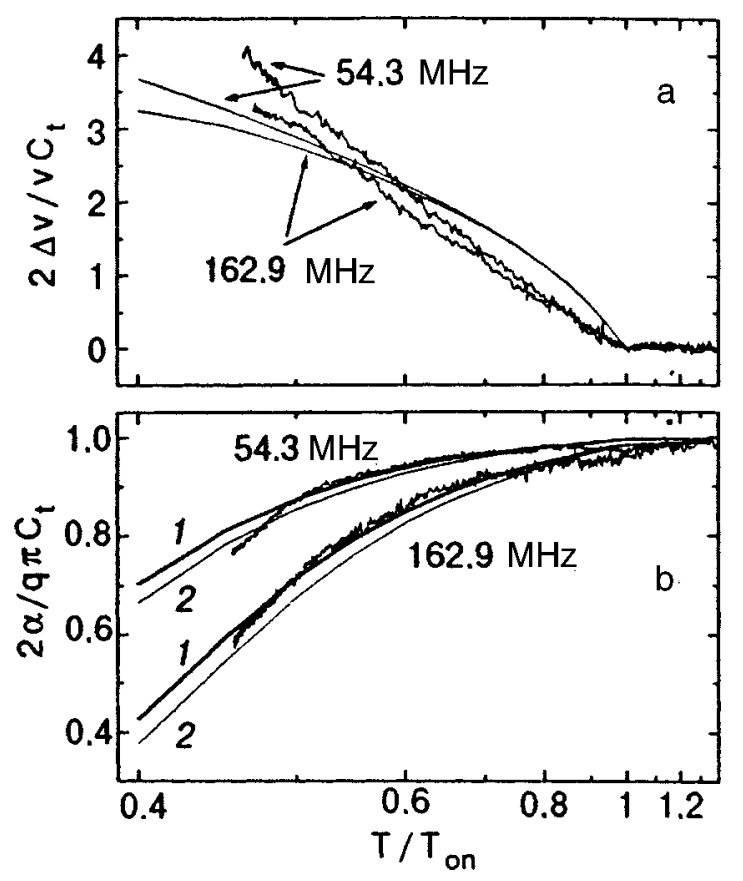

FIG. 2. Difference of the relative changes in the transverse sound velocity in the superconducting and normal states as a function of reduced temperature. Smooth curves correspond to the results of calculation $(\eta=0.85)(\mathrm{a})$; absorption in the superconducting phase: $\eta=0.89$ (curve 1) and 0.81 (curve 2) (b). The temperature is given on reciprocal scale.

taking into account magnetic scattering effects which eliminate the divergence in the derivatives of the order parameter and energy gap at $T_{c},{ }^{8}$ but a rigorous theory of interaction between sound and TLS in the presence of magnetic impurities has not yet been constructed. The assumption concerning the equality of the resonant contributions in the $s c$ - and $n$-phases might be incorrect due to a considerable narrowing of the tunnel levels upon a decrease in $\left(\tau_{\min }^{-1}\right)_{e s c},{ }^{9}$ which can affect the behavior of $\Delta v / v$ at lower temperatures, e.g., can change the normalization of the ordinate axis in Fig. 2a.

Figure $2 \mathrm{~b}$ shows the temperature dependences of variations in sound absorption, which is also normalized by $\pi C_{t} / 2$ in accordance with (2). Further, the normalized absorption in the normal phase was assumed to be equal to unity in conformity with (2) since the real value of $\alpha$ cannot be determined in the temperature interval under investigation (this can be done only from the difference in absorption in the normal and deep superconducting states). The theoretical dependences in the BCS model correctly reproduce the difference in the behavior of absorption at two frequencies, although the steepness of the variation of $\alpha / q$ for $T / T_{\text {on }} \sim 0.45$ is higher than the theoretical value. It should be recalled, however, that the steepness of experimental dependences in Fig. $2 \mathrm{~b}$ is also determined by the choice of the normalization constant $C_{t}$. The estimated value of $\eta \approx 0.85$ naturally depends on the value of $\Delta_{s c}$ : the value of $\eta$ decreases with $\Delta_{s c}$. In this case, theoretical dependences become still more gently sloping, and the estimated value of $\eta$ can not be reduced by a factor of more than two for the same normalization constant $C_{t}$.

Let us now estimate the TLS density of states. This can be done if we know the crossover temperature $T_{\text {cr }}$ at which the electron relaxation time becomes equal to the phonon one: $\left(\tau_{e}\right)_{\min }=\left(\tau_{\mathrm{ph}}\right)_{\min }$. Usually it is assumed that the crossover occurs at a temperature at which the velocity of sound passes through its maximum. ${ }^{3}$ In our case, $T_{\text {cr }} \approx 3.3 \mathrm{~K}$. The phonon relaxation rate is mainly determined by transverse phonons: ${ }^{3}$

$$
\left(\tau_{\min }^{-1}\right)_{\mathrm{ph}}=\frac{8 \gamma_{t}^{2}}{\pi \rho v_{t}^{5}} T^{3} .
$$

Combining (1), (3), and (5), we obtain

$$
\bar{P}=8.4\left(T_{\mathrm{cr}} / \eta\right)^{2} C_{t} \cdot 10^{32} \mathrm{erg}^{-1} \cdot \mathrm{cm}^{3}
$$

(in this equation, $T_{\mathrm{cr}}$ is in degrees). Substituting $T_{\mathrm{cr}}=3.3 \mathrm{~K}$, $\eta=0.85, \quad C_{t}=1.42 \cdot 10^{-5}, \quad$ we obtain $\quad \bar{P}=1.8 \cdot 10^{29}$ $\mathrm{erg}^{-1} \cdot \mathrm{cm}^{-3}$. This value is two-three orders of magnitude smaller than values known from literature. ${ }^{4}$

In formula (6), we specially singled out the quantities whose values are not known exactly. In actual practice, a noticeable contribution (proportional to $T^{2}$ ) to the velocity of sound is observed in the sample under investigation (we are planning to publish the corresponding results later); the combination of this contribution with the logarithmic dependence can also lead to the formation of a velocity peak. For this reason, the value of $T_{\text {cr }}$ can be slightly higher. The fitting value of $\eta$ (see Fig. 2b) depends on the energy gap for which the BCS value was used. If our assumption concerning the effect of magnetic scattering is confirmed, the energy gap will be smaller, ${ }^{8}$ which can reduce the estimated value of $\eta^{2}$ by a factor of several times. The exact value of $C_{t}$ is not known to us either; it can be obtained from the logarithmic decrease in the velocity of sound in the deep superconducting state. On account all these remarks, the estimated value of $\bar{P}$ in the alloy under investigation can be an order of magnitude higher than the value given above, but still considerably smaller than the known values from the literature sources.

In summary, the values of the TLS density of states in the amorphous alloy $\mathrm{Zr}_{41.2} \mathrm{Ti}_{13.8} \mathrm{Cu}_{12.5} \mathrm{Ni}_{10} \mathrm{Be}_{22.5}$ estimated on the basis of acoustic measurements are much smaller than for other metglasses. This is probably due to the very low rate of cooling of the melt $(\sim 10 \mathrm{~K} / \mathrm{s})$. The observed peculiarities of superconducting ordering indicate, on one hand, the twophase structure of the amorphous alloy under investigation, and on the other hand, the possible realization of a gapless superconductivity in this material in a narrow temperature range.

The authors are grateful to V. Z. Bengus, E. D. Tabachnikova, and A. S. Bakai for fruitful discussions and to E. A. Masalitin for his assistance in preparing the apparatus.

*E-mail: fil@ilt.kharkov.ua

\footnotetext{
${ }^{1}$ P. W. Anderson, B. I. Halperin, and C. M. Varma, Philos. Mag. 25, 1 (1972).

${ }^{2}$ W. A. Phillips, J. Low Temp. Phys. 7, 351 (1972)

${ }^{3}$ S. Hunklinger and A. K. Raychaudhuri, Progr. Low Temp. Phys. 9, 266 (1986).

${ }^{4}$ D. A. Parshin, Phys. Rev. B 49, 9400 (1994).
} 
${ }^{5}$ A. Pecker and W. L. Johnson, Appl. Phys. Lett. 63, 2342 (1993).

${ }^{6}$ J. L. Black and P. Fulde, Phys. Rev. Lett. 43, 453 (1979).

${ }^{7}$ A. Gerber, A. Milner, A. Voronel et al., Physica C 275, 333 (1997).

${ }^{8}$ A. A. Abrikosov and L. P. Gor'kov, Zh. Éksp. Teor. Fiz. 39, 1781 (1960)
[ Sov. Phys. JETP 12, 1243 (1960)].

${ }^{9}$ N. Thomas, Philos. Mag. B 48, 297 (1983).

Translated by R. S. Wadhwa 